\title{
Electronic properties of wurtzite GaAs: A correlated structural, optical, and theoretical analysis of the same polytypic GaAs nanowire
}

\author{
Alexander Senichev ${ }^{1, t, \S}(\varangle)$, Pierre Corfdir ${ }^{2, \ddagger, \S}$, Oliver Brandt ${ }^{2}$, Manfred Ramsteiner ${ }^{2}$, Steffen Breuer ${ }^{2, \|}$, \\ Jörg Schilling ${ }^{3}$, Lutz Geelhaar ${ }^{2}$, and Peter Werner ${ }^{1}$ \\ ${ }^{1}$ Max-Planck-Institut für Mikrostrukturphysik, Halle 06120, Germany \\ ${ }^{2}$ Paul-Drude-Institut für Festkörperelektronik, Berlin 10117, Germany \\ ${ }^{3}$ Centre for Innovation Competence SiLi-nano, Martin-Luther-Universität, Halle 06120, Germany \\ ${ }^{\dagger}$ Present address: Birck Nanotechnology Center, Purdue University, West Lafayette, Indiana 47907, USA \\ ₹ Present address: Fraunhofer HHI, Berlin 10587, Germany \\ ${ }^{\square}$ Present address: ABB Corporate Research, Baden-Dättwil 5405, Switzerland \\ ${ }^{\S}$ Alexander Senichev and Pierre Corfdir contributed equally to this work.
}

Received: 5 August 2017

Revised: 15 March 2018

Accepted: 17 March 2018

(c) The author(s) 2018. This article is published with open access at link.Springer.com

\section{KEYWORDS}

nanowires, crystal-phase quantum structures, wurtzite GaAs, strain, near-field scanning optical microscopy, photoluminescence

\begin{abstract}
III-V compound semiconductor nanowires are generally characterized by the coexistence of zincblende and wurtzite structures. So far, this polytypism has impeded the determination of the electronic properties of the metastable wurtzite phase of GaAs, which thus remain highly controversial. In an effort to obtain new insights into this topic, we cross-correlate nanoscale spectral imaging by near-field scanning optical microscopy with a transmission electron microscopy analysis of the very same polytypic GaAs nanowire dispersed onto a Si wafer. Thus, spatially resolved photoluminescence spectra could be unambiguously assigned to nanowire segments whose structure is known with lattice-resolved accuracy. An emission energy of $1.528 \mathrm{eV}$ was observed from extended zincblende segments, revealing that the dispersed nanowire was under uniaxial strain presumably due to interaction with its supporting substrate. These crucial information and the emission energy obtained for extended pure wurtzite segments were used to perform envelope function calculations of zincblende quantum disks in a wurtzite matrix as well as the inverse structure. In these calculations, we varied the fundamental bandgap, the electron mass, and the band offset between zincblende and wurtzite GaAs. From this multi-parameter comparison with the experimental data, we deduced that the bandgap between the $\Gamma_{8}$ conduction and A valence band ranges from 1.532 to $1.539 \mathrm{eV}$ in strain-free wurtzite GaAs, and estimated values of 1.507 to $1.514 \mathrm{eV}$ for the $\Gamma_{7}-\mathrm{A}$ bandgap.
\end{abstract}

Address correspondence to senichev@purdue.edu 


\section{Introduction}

When III-V compound semiconductors are grown in the form of nanowires, their crystal lattice may adopt a wurtzite (WZ) structure, although their thermodynamically stable crystal phase has, with the exception of nitrides, a zincblende (ZB) structure [1]. Bulk crystals and planar thin films of these materials invariably crystallize in the zincblende structure. However, for nanowires extending along the $\langle 111\rangle$ direction, the formation probability of the different crystal phases is generally similar, and the preferential formation of a specific phase during the nucleation of each atomic layer sensitively depends on several energetic and kinetic factors, such as the supersaturation of the catalyst, interfacial energies, and the nucleation site [2-5]. Changes of the stacking sequence are, hence, easily introduced, and such nanowires consequently represent axial polytype heterostructures.

Since the different polytypes have different electronic band structures [6], the resulting polytype heterostructures give rise to complex luminescence spectra [7-9] and can affect charge transport [10,11]. On the one hand, such phenomena may deteriorate the performance of nanowire devices. On the other hand, crystal-phase quantum structures may also be exploited as the very basis for fundamental investigations and applications since they are structurally perfect by nature. More specifically, such heterostructures exhibit interfaces that are defined by crystal stacking and are hence atomically abrupt. Furthermore, crystal-phase heterostructures are free of any alloy disorder, in contrast to conventional heterostructures based on changes in chemical composition that often involve ternary materials.

Impressive progress has been achieved with respect to the understanding and control of the formation of crystal polytypes in nanowires [5, 12, 13]. Equally important is the understanding of the electronic structure of crystal-phase quantum structures. This objective primarily requires the determination of the WZ band structure, since the properties of the ZB phase are very well known. Most studies in this direction have been carried out on the polytypic III-V compound semiconductor GaAs. Despite considerable efforts, the results from the vast body of literature on this subject are entirely inconsistent [9, 14-24]. For example, the reported values for the bandgap of WZ GaAs scatter from $20 \mathrm{meV}$ below [14] to $100 \mathrm{meV}$ above [17] the bandgap of ZB GaAs. To the best of our knowledge, the results of all reported studies to date are summarized in Fig. S1 in the Electronic Supplementary Material (ESM). This overview reveals that even among recent studies, there is no consensus on the band structure of WZ GaAs. Its experimental analysis is made challenging by the fact that bulk material is essentially not available, as reported by McMahon and Nelmes [25]; moreover, in nanowires, typically both phases occur on a nanometer length scale, leading to difficulties in assigning the optical transitions to specific structural configurations. The diffusion length in GaAs nanowires is on the order of $1 \mu \mathrm{m}$ [26], which makes it complicated to characterize WZ GaAs nanowires by standard luminescence spectroscopy techniques. This requires either WZ GaAs nanowires with a stacking faults/twins density much lower than 1 per micrometer, which in most cases poses a challenge, or highly spatially-resolved spectroscopic techniques.

In the present study, we characterized one and the same dispersed $\mathrm{GaAs} /(\mathrm{Al}, \mathrm{Ga}) \mathrm{As}$ core/shell nanowire by both near-field scanning optical microscopy (NSOM) and transmission electron microscopy (TEM). Thus, we established a cross-correlation between photoluminescence bands and crystal structure with extreme spatial resolution. From these experiments, we succeeded at extracting the emission energy at $10 \mathrm{~K}$ of extended $\mathrm{ZB}$ and $\mathrm{WZ}$ segments, $\mathrm{WZ}$ quantum disks in a ZB matrix, and ZB quantum disks in a $W Z$ matrix. The results for the extended $\mathrm{ZB}$ segment revealed that the nanowire was actually under a compressive uniaxial strain along the nanowire axis, presumably due to its interaction with the supporting substrate. A homogeneous uniaxial strain naturally affects the electronic transitions in all other parts of a nanowire [27], and it is a factor that has so far not been taken into account in the analysis of dispersed GaAs nanowires. The advantage of our study is that we obtained cross-correlated experimental data for different well-defined structural configurations in the same nanowire. We compared this set of data with the results of envelope function calculations in which we varied WZ band structure parameters. On the basis of this multi-parameter comparison, we concluded that in strain-free WZ GaAs, the fundamental bandgap occurs between the $\Gamma_{8}$ conduction and $A$ valence bands, and that the bandgap between the $\Gamma_{8}$ 
conduction and A valence band ranges between 1.532 and $1.539 \mathrm{eV}$.

\section{Results and discussion}

Figure 1 shows a hyper-spectral map taken on the $\mathrm{GaAs} /(\mathrm{Al}, \mathrm{Ga})$ As core/shell nanowire under investigation by using NSOM at $10 \mathrm{~K}$. The positions of the end facets of the nanowire, deduced from a topographical image (not shown), are highlighted by black arrows. The spatial distributions of the photoluminescence (PL) peak intensity and energy shown in Figs. 1(a) and 1(b), respectively, both reveal domains of various lengths along the nanowire (NW) axis. The locations of the change in PL energy coincide with abrupt changes in PL peak intensity, as emphasized by the vertical dashed lines. These domains of constant PL energy and intensity along the nanowire axis can be as short as $270 \mathrm{~nm}$ or extend over more than $1 \mu \mathrm{m}$. Small variations in PL peak energy are also observed perpendicular to the nanowire axis (Fig. 1(b)). They arise from the fact that when moving the near-field probe away from the axis of the wire, the PL intensity decreases drastically, making the determination of the PL peak energy less accurate. It should also be noted that the PL intensity from the tip of the nanowire is much lower than the one detected along the nanowire length, which is likely due to the simultaneous unintentional axial growth of $(\mathrm{Al}, \mathrm{Ga}) \mathrm{As}$ during the radial growth stage (Fig. 2(b), below $1.5 \mu \mathrm{m})$.

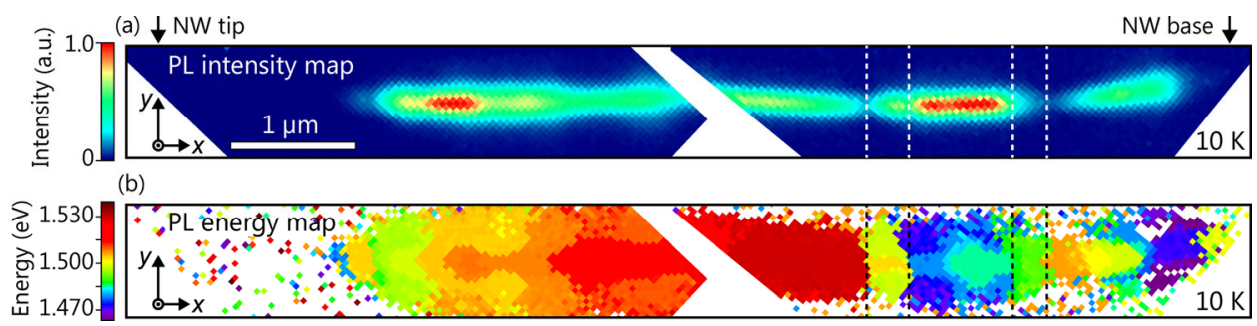

Figure 1 Near-field spectral imaging of a single GaAs nanowire comprising a mixture of zincblende (ZB) and wurtzite (WZ) crystal phases of different order and lateral extent. All optical measurements were performed at a temperature of $10 \mathrm{~K}$. (a) Two-dimensional spatial map of the PL intensity of the most intense peak selected at each pixel of the image. The color code indicates the normalized PL intensity distribution in the area containing the nanowire of interest. (b) Two-dimensional spatial map of the PL peak energy of the most intense peak in the spectrum of a given pixel. The color code denotes the emission energy distribution. The scale bar of $1 \mu \mathrm{m}$ is common for both maps.

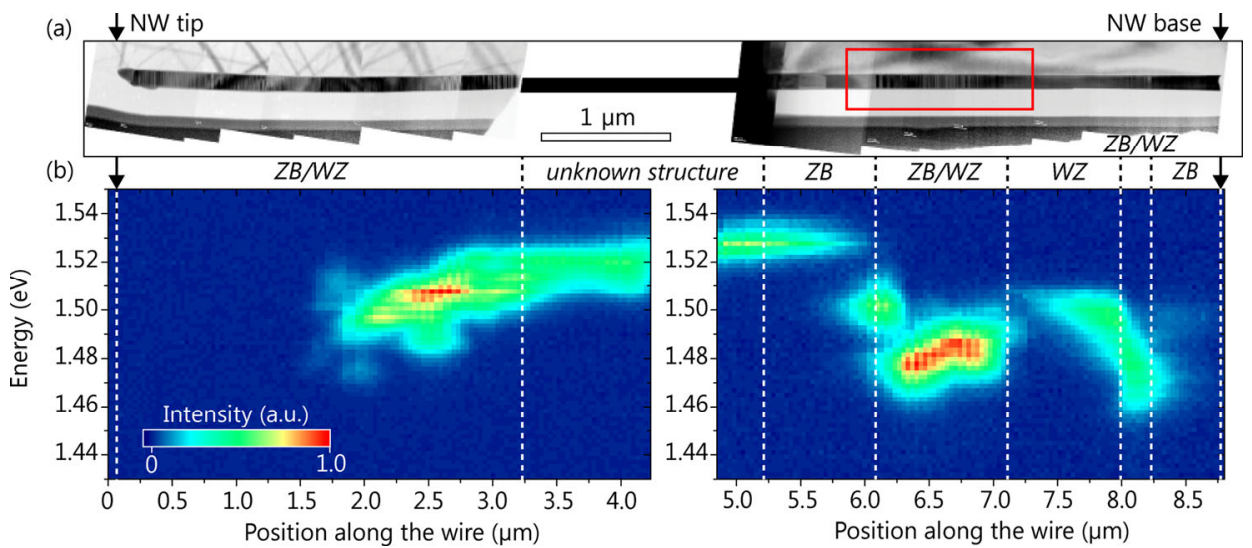

Figure 2 (a) A set of diffraction contrast TEM micrographs acquired along the whole length of the wire. The middle part is cut due to the FIB preparation of the TEM sample. Dashed lines indicate interfaces between the nanowire segments with a virtually defect-free ZB crystal structure, disordered superlattices of the ZB and WZ phases, and defect-free WZ crystal structure. The crystal phase structure was analyzed using lattice-resolved HAADF-STEM micrographs (not shown here). (b) Two-dimensional map of the spatial and spectral distribution of the photoluminescence emission acquired by NSOM. Photoluminescence spectra were taken from central pixels selected along the wire (Fig. 1(a)). The vertical axis indicates the photoluminescence energy and covers the energy range between 1.43 and 1.55 $\mathrm{eV}$, which includes all detected spectral features. The horizontal axis indicates the position of each local PL spectrum along the nanowire. The white dashed lines on the spatial-spectral map highlight regions in correspondence to the classification of the crystal structure. The scale bar of $1 \mu \mathrm{m}$ applies to both the TEM map and the spectral images. 
While the short segments with constant PL energy and intensity exhibit PL energies below $1.515 \mathrm{eV}$, i.e., below the recombination energy of free excitons in strain-free ZB GaAs at $10 \mathrm{~K}[28,29]$, we observe energies above the bandgap of strain-free $\mathrm{ZB} \mathrm{GaAs}$ for a long segment located in the middle part of the wire. To relate the PL energy with the local microstructure of the nanowire, we have performed transmission electron microscopy (TEM) on the same nanowire. We emphasize that the direct correlation between optical and structural properties is made possible by the fact we operate our NSOM experiment in an illumination-collection geometry (see Methods). In this geometry, the emission is collected from the same spot that was excited by the near-field probe, which we use for both local excitation and local detection. Such a study in a standard PL experiment and cathodoluminescence measurements with local excitation and global detection would be hindered by the diffraction limited spatial resolution and/or the diffusion of excitons along the nanowire axis. Figure 2(a) shows the result of overlapping TEM micrographs acquired along the whole nanowire. The missing $1.5 \mu \mathrm{m}$ long segment in the middle of the nanowire was cut during the lift-off process by focused ion beam (FIB) and was therefore not available for the TEM analysis. The remaining lamellae contain two wire segments with a length of about 3 and $4 \mu \mathrm{m}$. The dark and bright stripes observed in Fig. 2(a) along the nanowire axis originate from rotationally twinned ZB segments or $\mathrm{WZ}$ inclusions that produce different electron diffraction conditions [30].

The quantitative analysis of the change in crystal structure along the nanowire axis shown in Fig. 2(a) has been obtained by high-angle annular dark-field (HAADF) scanning TEM (STEM). As displayed in Fig. 2(a), we can identify three different types of nanowire segments: $\mathrm{ZB} \mathrm{GaAs} \mathrm{segments} \mathrm{that} \mathrm{are} \mathrm{virtually} \mathrm{free}$ of twins $\left(<3\right.$ twins $\left.\mu \mathrm{m}^{-1}\right), \mathrm{ZB} / \mathrm{WZ}$ segments showing alternating $\mathrm{ZB}$ and $\mathrm{WZ}$ domains of various lengths, and $W Z$ segments that are free of stacking faults. The interface between the $\mathrm{ZB}$ and $\mathrm{ZB} / \mathrm{WZ}$ regions is taken at the appearance of the first rotationally twinned $\mathrm{ZB}$ segment, and that between the $\mathrm{ZB} / \mathrm{WZ}$ and $\mathrm{WZ}$ regions at the emergence of the last thin $Z B$ inclusion in the WZ lattice.

The evolution of the local near-field PL spectra along the nanowire axis is shown in Fig. 2(b). As highlighted by the white dashed lines, the transitions in the crystal structure from $\mathrm{ZB}$ to $\mathrm{ZB} / \mathrm{WZ}$ and from $\mathrm{WZ}$ to $\mathrm{ZB} / \mathrm{WZ}$ result in abrupt changes in the PL spectrum. Figures 3(a) and 3(b) show typical HAADFSTEM micrographs taken on the $\mathrm{ZB}$ and $\mathrm{WZ}$ regions, respectively. Representative near-field PL spectra acquired for these regions are displayed in Figs.3(c) and $3(\mathrm{~d})$, respectively. Since the near-band edge part of the low-temperature PL spectra of GaAs is usually dominated by free excitons, these spectra indicate that the energy of free excitons in the $\mathrm{WZ}$ and $\mathrm{ZB}$ segments of this GaAs nanowire is 1.506 and $1.528 \mathrm{eV}$, respectively. Surprisingly, the nanowire segment giving rise to a PL line at an energy higher than the bandgap of strain-free ZB GaAs $(1.519 \mathrm{eV})$ exhibits the ZB crystal structure (Figs. 3(a) and 3(c)). Note that the energy of this line is constant to within $1 \mathrm{meV}$ over the entire length $(800 \mathrm{~nm})$ of the $\mathrm{ZB}$ segment (see Fig. 2(b)). The PL spectrum at the position of the second $\mathrm{ZB}$ segment near the base of the nanowire exhibits two emission lines at energies of 1.470 and $1.495 \mathrm{~V}$, respectively. The low-energy peak originates from a disordered mixture of thin $\mathrm{ZB}$ and $\mathrm{WZ}$ inclusions in the adjacent $\mathrm{ZB} / \mathrm{WZ}$ segment. Its intensity notably decreases when the near-field probe moves away towards the end of the nanowire. The weak high-energy peak at $1.495 \mathrm{eV}$ may arise from carbon-related bandacceptor transitions that were also observed in previous work by Jahn et al. [9]. Note that the PL intensity near the base of the nanowire is drastically reduced due to the diffusion of carriers to the end of the nanowire and their subsequent non-radiative recombination.

Obviously, the observation of an excitonic transition at $1.528 \mathrm{eV}$ in $\mathrm{ZB} \mathrm{GaAs}$ is inconsistent with the wellknown bandgap and free-exciton transition energy $(1.515 \mathrm{eV})$ for strain-free ZB GaAs at low temperature. We have excluded possible artifacts that could result in a blueshift of the exciton line by extensive test experiments that are described in more detail in the ESM (Figs. S2 and S3 in the ESM). The only remaining explanation for the measured transition energy is that the studied nanowire is strained. The lattice mismatch between the $\mathrm{GaAs}$ core and $\mathrm{Al}_{0.1} \mathrm{Ga}_{0.9} \mathrm{As}$ shell of the nanowires studied in this work is as small as $0.0138 \%$ (details are given in the ESM). Assuming that the core and shell share this mismatch according 

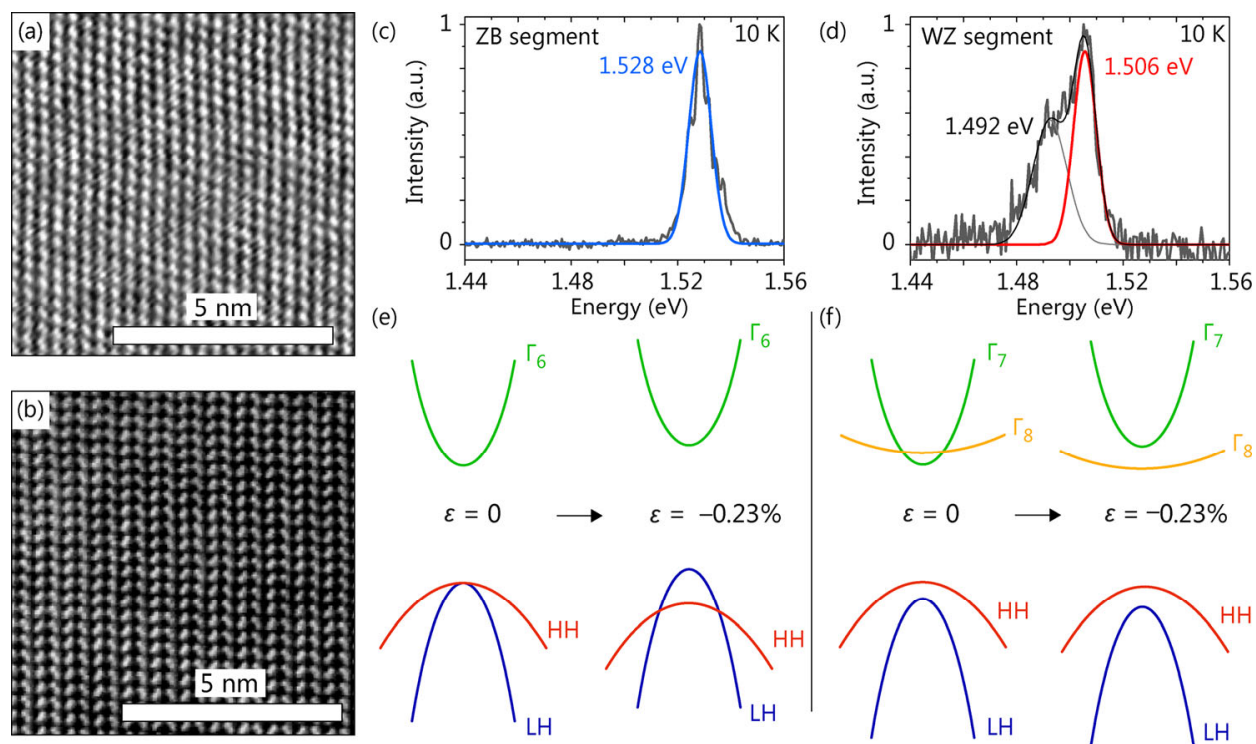

Figure 3 (a) and (b) Representative high-resolution HAADF-STEM micrographs of the ZB (a) and WZ (b) nanowire segments. (c) and (d) Representative local PL spectra of the ZB (c) and WZ (d) regions recorded at $10 \mathrm{~K}$. The energy of the PL band at the highest energy has been obtained using a Gaussian fit (solid line), yielding a free exciton energy of 1.528 and $1.506 \mathrm{eV}$ for the ZB and WZ nanowire segments, respectively. The peak at $1.492 \mathrm{eV}$ in panel $(\mathrm{d})$ arises from an emission from the neighboring $\mathrm{ZB} / \mathrm{WZ}$ region, i.e., the region with a mixture of thin $\mathrm{ZB}$ and $\mathrm{WZ}$ segments of different order and lateral extent. (e) Band structure of $\mathrm{ZB}$ GaAs without strain $(\varepsilon=0)$ and with a uniaxial strain of $\varepsilon=-0.23 \%$ along the nanowire axis. The strain leads to an increase in the bandgap and to a change in the character of the lowest valence band from heavy hole (HH) to light hole (LH). (f) Band structure of WZ GaAs with and without uniaxial strain of $\varepsilon=-0.23 \%$ along the nanowire axis. The strain leads to a change in the symmetry of the lowest conduction band from $\Gamma_{7}$ to $\Gamma_{8}$. The energy of the $\Gamma_{8}-\mathrm{A}$ transition for $\varepsilon=-0.23 \%$ is lower than that of the $\Gamma_{7}-\mathrm{A}$ one in strain-free material.

to their cross-sectional area, the strain induced by the shell is not more than $0.01 \%$, a negligibly small value [31,32]. Moreover, the core should experience a tensile axial strain from the shell, which would cause a red-shift instead of the observed blue-shift [32]. As discussed in references [33, 34] for $\mathrm{GaN}$ nanowires, nanowires dispersed on a supporting substrate may become strained, resulting in a shift and broadening of the lines in PL spectra. This strain can, for instance, arise from a mismatch between the thermal expansion coefficients of the nanowire and substrate [33], bending of the nanowire introduced during dispersion [34], or the presence of solvent residues that efficiently bond the dispersed nanowires to the substrate [35]. In the absence of any notable bending of the studied nanowire, as evidenced by both optical and scanning electron microscopy (Fig. S4 in the ESM), we assume that the $13 \mathrm{meV}$ blueshift of the free exciton transition in the $\mathrm{ZB}$ segments of the nanowire can be due to the presence of a uniaxial compressive strain along the nanowire axis. Using the deformation potential for ZB GaAs reported in Ref. [36], we deduce from the blueshift of the free exciton that the uniaxial strain along $\langle 111\rangle$ is $\varepsilon=-0.23 \%$. It should be noted that for this strain state, the fundamental hole in $\mathrm{ZB}$ GaAs is the light hole $(\mathrm{LH})$, as schematically depicted in Fig. 3(e).

The evolution of the band structure of WZ GaAs under uniaxial strain along $\langle 0001\rangle$ has been previously studied both theoretically [37] and experimentally [27]. These studies showed that a uniaxial strain along the $\langle 0001\rangle$ direction results in a crossing of the $\Gamma_{7}$ and $\Gamma_{8}$ conduction bands in WZ GaAs [27,37]. The exact value of the strain at which this crossing occurs is much less clear, but both theoretical $[37,38]$ and experimental [27] data favor a situation in which the conduction-band minimum is of $\Gamma_{7}$ symmetry at zero strain, and experiment indicates that the bands cross for a compressive uniaxial strain as small as $-0.12 \%$.

Assuming that the uniaxial strain of $\varepsilon=-0.23 \%$ is uniform and is thus experienced by all segments in the nanowire, the valence-band maximum in $\mathrm{WZ}$ GaAs is still formed by the heavy hole A band. With the deformation potentials reported in Ref. [27], the $\Gamma_{7}-\mathrm{A}$ bandgap is blueshifted by $28.8 \mathrm{meV}$ (Fig. 3(f)), 
while the $\Gamma_{8}-\mathrm{A}$ bandgap is redshifted by $20.4 \mathrm{meV}$ for $\varepsilon=-0.23 \%$. Since the free exciton energy measured for the strained WZ GaAs nanowire segment is $1.506 \mathrm{eV}$ (Fig. 3(d)), we conclude that the $\Gamma_{8}-A$ excitonic bandgap of strain-free $\mathrm{WZ} \mathrm{GaAs}$ at $10 \mathrm{~K}$ is thus $1.526 \mathrm{eV}$. The observation of intense emission bands involving $\Gamma_{8}$ electron states seems at first to be in contradiction with the negligibly small matrix elements predicted for the $\Gamma_{8}-\mathrm{A}$ optical transitions at the center of the Brillouin zone [6]. However, as discussed in Ref. [39], the $\Gamma_{8}-\mathrm{A}$ matrix elements increase with increasing the $k$ vector. We thus infer that it is possible to detect intense transitions from WZ GaAs due to the finite extent of the wavefunction of the $\Gamma_{8}-\mathrm{A}$ exciton in the $k$-space. However, we can neither rule out that the transition observed in the WZ segment stems from excitons bound to impurities, which have a highoscillator strength even in indirect semiconductors. At the same time, we note that bound excitons in GaAs are very shallow states with a binding energy on the order of 1-2 meV. Thus, in the following we assume for simplicity free excitons and emphasize that all quantitative conclusions would be negligibly affected.

In contrast to these pure $\mathrm{WZ}$ and $\mathrm{ZB}$ segments, whose detected PL lines presumably originate from the free exciton recombination, the transitions from mixed $\mathrm{ZB} / \mathrm{WZ}$ segments arise from excitons localized at the crystal phase quantum structures $[8,14]$. Specifically, the band alignment between $\mathrm{WZ}$ and $\mathrm{ZB}$ GaAs is type II, and recombination in the $\mathrm{ZB} / \mathrm{WZ}$ segments involves electrons and holes confined in segments with $\mathrm{ZB}$ and $\mathrm{WZ}$ crystal structures, respectively. As a result of this localization, the PL energy for the mixed $\mathrm{ZB} / \mathrm{WZ}$ segments of the nanowire ranges between 1.477 and $1.512 \mathrm{eV}$, which are values well below the excitonic bandgap of the strained ZB GaAs segment (Fig. 2(b)). The region highlighted by the red rectangle in Fig. 2(a) is of particular interest. As shown by the enlarged diffraction-contrast TEM micrograph in Fig. 4(a), this nanowire segment is a 1- $\mu \mathrm{m}$-long transition region between the twin-free ZB GaAs and stacking fault-free WZ GaAs, where the change in the crystal structure occurs by a gradual increase in the fraction of the WZ phase. A NSOM spectral mapping taken on the same nanowire segment is displayed in Fig. 4(b). Clearly, the transition region encompasses three domains $\mathrm{A}, \mathrm{B}$,

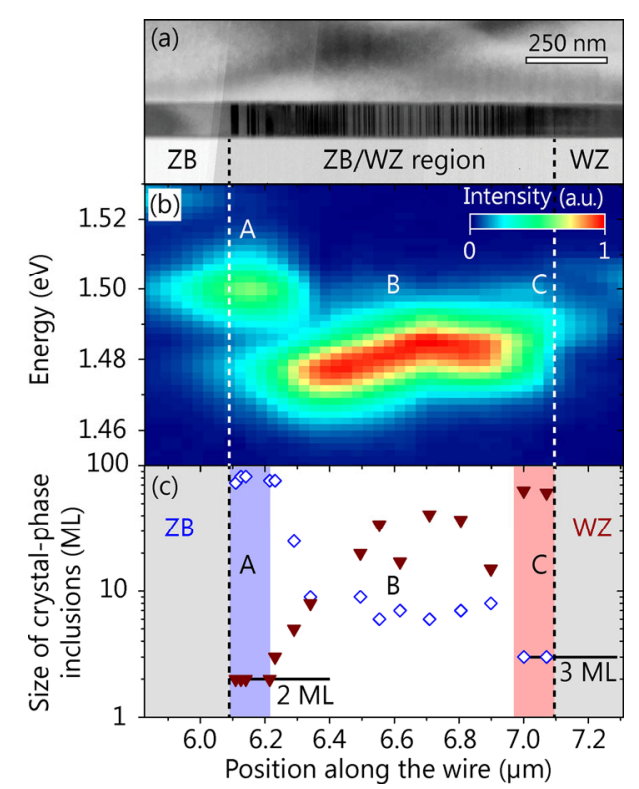

Figure 4 (a) Enlarged diffraction contrast TEM micrograph of the area marked in Fig. 2(a) (red rectangle). The crystal structure changes from a pure $\mathrm{ZB}$ to a pure $\mathrm{WZ}$ phase by a gradual increase of the $\mathrm{WZ}$ fraction ( $\mathrm{ZB} / \mathrm{WZ}$ region). Dashed lines indicate the appearance of the first $W Z$ disk in the $Z B$ region and the last $\mathrm{ZB}$ disk in the $\mathrm{WZ}$ region defining the interfaces with the $\mathrm{ZB} / \mathrm{WZ}$ region. (b) Spatially and spectrally resolved map of local PL spectra in the selected region of the wire. The vertical axis indicates the emission energy, which is scaled from 1.450 to $1.530 \mathrm{eV}$ to highlight all spectral features of this region. The horizontal axis shows the position of the corresponding local near-field PL spectra along the selected region. The spectral resolution of about $3 \mathrm{meV}$ is defined by the settings of the monochromator. The spatial resolution of the NSOM hyperspectral imaging is of the order of $150 \mathrm{~nm}$, which is in good agreement with the previously reported values for uncoated fiber-based near-field probes [56]. (c) Thickness of the longest ZB (open symbols) and WZ (full triangles) segments in each high-resolution STEM micrograph acquired along the $\mathrm{ZB} / \mathrm{WZ}$ region.

and $C$ with distinct PL peak energies: the PL energy is $1.501 \mathrm{eV}$ in domain $\mathrm{A}$, it continuously increases from 1.477 to $1.485 \mathrm{eV}$ in domain $\mathrm{B}$, and it is equal to $1.492 \mathrm{eV}$ in domain $\mathrm{C}$.

A quantitative analysis of the high-resolution HAADFSTEM micrographs of this transition region is shown in Fig. 4(c). In order to account for the change in the $\mathrm{WZ}$ phase fraction, we plot in this figure the evolution of the length of the longest $\mathrm{ZB}$ and WZ segments measured for each TEM micrograph as a function of the position of the micrograph along the NW axis. The nanowire segments with defect-free $\mathrm{ZB}$ and $\mathrm{WZ}$ structure are shown in grey color on the left and right-hand 
sides of the transition region, respectively. Domain $\mathrm{A}$ is a $280 \mathrm{~nm}$ long ZB GaAs segment exhibiting a sequence of rotational twin boundaries, which can be described by 2 monolayers (MLs) of WZ GaAs separated by ZB GaAs segments with a length between 60 and $80 \mathrm{ML}$, i.e., about $15-20 \mathrm{~nm}$. Due to the type II band alignment between $\mathrm{WZ}$ and $\mathrm{ZB}$ GaAs, the holes are localized in the WZ GaAs disk and the electrons are maintained in the neighboring $\mathrm{ZB}$ segment in the vicinity of the WZ-disk by the attractive Coulomb interaction exerted by the hole [40]. With regard to domain $C$, we observe $\mathrm{I}_{1}$ stacking faults in WZ GaAs, which can be represented by $3 \mathrm{ML}$ thick disks of ZB GaAs separated by WZ segments with a thickness of about 60 MLs. The electron wavefunction of the exciton is confined in the $\mathrm{ZB}$ disk, while the hole is repelled into the $\mathrm{WZ}$ barrier and maintained in the vicinity of the $\mathrm{ZB}$ disk by the Coulomb interaction with the electron. Finally, domain $\mathrm{B}$ is much longer than domains $\mathrm{A}$ and $\mathrm{C}$ $(700 \mathrm{~nm})$ and consists of alternating segments of $\mathrm{ZB}$ and WZ GaAs of various widths, i.e., it is a disordered polytype superlattice. Since changes in the thickness between adjacent WZ and ZB segments lead to a variation in the confinement energies, the varying $P L$ energy in region B observed in Fig. 4(b) is a direct consequence of the change in the WZ phase fraction along the nanowire length.

Due to the strong structural disorder, only qualitative information can be obtained from the evolution of the PL energy within domain B. In contrast, domains $A$ and $C$ are of direct interest, since in both the density of crystal-phase structures is low. Figure 5(a) shows a PL spectrum taken on domain A at $10 \mathrm{~K}$. The spectrum is dominated by a band centered at $1.501 \mathrm{eV}$. A weaker and broader band at $1.477 \mathrm{eV}$ is also detected. Figure 5(b) displays an enlarged diffraction-contrast TEM micrograph of domain $\mathrm{A}$. The alternating dark and bright stripes in the micrograph arise from the rotationally twinned ZB segments. This assignment is verified by the lattice-plane resolved HAADF-STEM micrograph of the interface between two rotationally twinned ZB segments shown in Fig. 5(c), which also confirms that the interface between
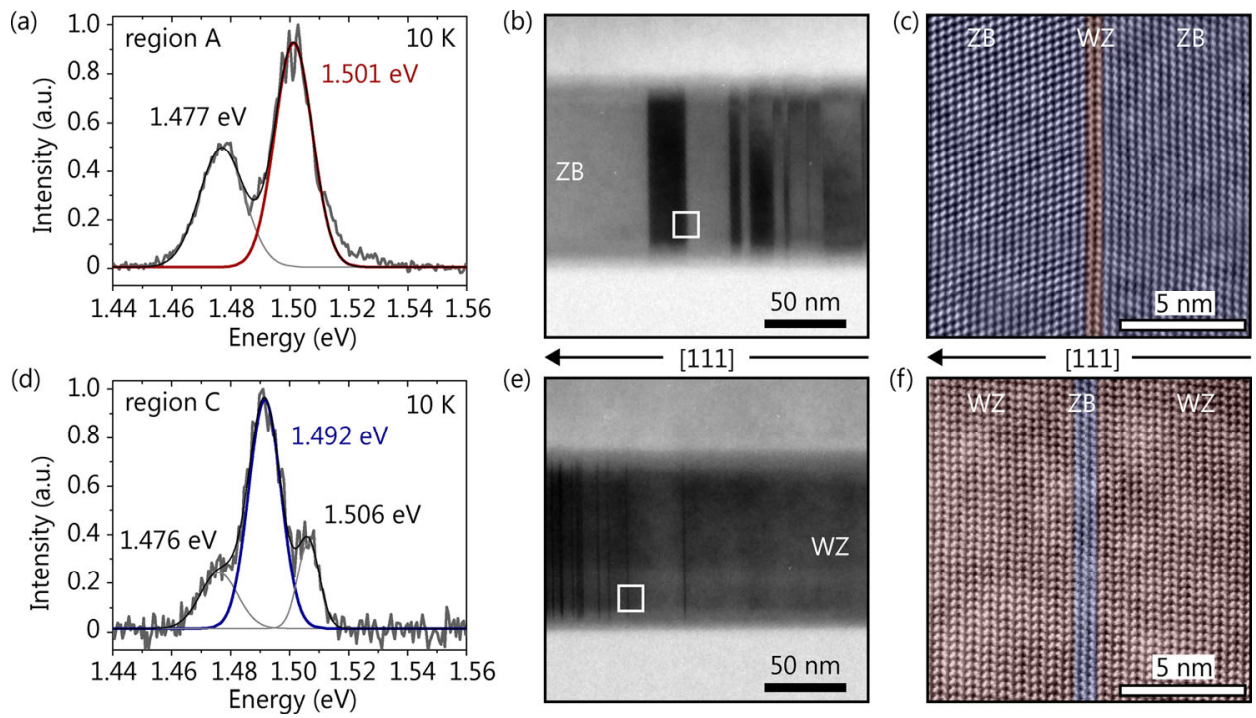

Figure 5 (a) Representative local PL spectrum selected from domain A indicated in Fig. 4(b). The most intense PL band has an emission energy of $1.501 \mathrm{eV}$ as determined by the Gaussian fit (red solid line). The PL band at $1.477 \mathrm{eV}$ originates from domain B shown in Fig. 4(b). (b) Enlarged diffraction-contrast TEM micrograph of the same domain A. The alternating dark and bright stripes correspond to rotationally twinned ZB segments, which produce different electron diffraction conditions. (c) Representative high-resolution HAADF-STEM micrograph of the interface between two rotationally twinned segments highlighted by the white square in (b). The stacking order of the $\langle 111\rangle$ lattice planes at the interface produces a 2-ML-thick WZ inclusion. The rotationally twinned ZB segments are shown in blue and the single WZ inclusion in red, respectively. (d) Representative local PL spectrum from domain C indicated in Fig. 4(b). The emission is dominated by a transition at $1.492 \mathrm{eV}$ as is defined by the Gaussian fit (blue solid line). (e) Enlarged diffraction-contrast TEM micrograph of the same domain C. The sharp dark vertical lines correspond to the short inclusions of ZB segments in the WZ matrix. (f) Representative high-resolution HAADF-STEM micrograph of the 3-ML-thick ZB disk in the WZ matrix dominating in domain $\mathrm{C}$. The WZ segments are shown in red and the single ZB inclusion in blue, respectively.

INIIVRSITY PRESS SP Springer | www.editorialmanager.com/nare/default.asp 
two rotationally twinned segments can be seen as a 2 ML thick insertion of the WZ material in a ZB matrix [41-43]. The transition at $1.477 \mathrm{eV}$ is also detected in domain B (Fig. 4(b)). Therefore, we attribute its occurrence in domain $\mathrm{A}$ to the tail of the emission from domain $B$. The energy of the exciton in a 2 ML thick quantum well of WZ GaAs embedded in a ZB GaAs matrix in the presence of a uniaxial strain of $\varepsilon=-0.23 \%$ is thus $1.501 \mathrm{eV}$.

Domain $C$ is located at the opposite end of the mixed $\mathrm{ZB} / \mathrm{WZ}$ transition region and exhibits a low density of $\mathrm{I}_{1}$ stacking faults. Figure $5(\mathrm{~d})$ shows a PL spectrum of this domain recorded at $10 \mathrm{~K}$. The spectrum is dominated by a transition at $1.492 \mathrm{eV}$ with low and high energy shoulders at 1.476 and $1.506 \mathrm{eV}$, respectively. The diffraction-contrast TEM and lattice-plane resolved HAADF-STEM micrographs shown in Figs. 5(e) and $5(\mathrm{f})$, respectively, confirm that the $\mathrm{I}_{1}$ stacking faults in domain $\mathrm{C}$ consist of $3 \mathrm{ML}$ thick ZB disks. Similar to what is observed for domain A in Fig. 5(a), the shoulders at 1.476 and $1.506 \mathrm{eV}$ are due to the low intensity tails of the PL from domain $\mathrm{B}$ and from the stacking fault-free WZ GaAs segment, respectively (Fig. 3(d)). Therefore, we conclude from Fig. 5(d) that the energy of excitons bound to the uniaxially strained $\mathrm{I}_{1}$ stacking faults in WZ GaAs is $1.492 \mathrm{eV}$.

Next, we compare the energy measured for excitons bound to twin boundaries (i.e., $2 \mathrm{ML}$ thick $\mathrm{WZ}$ quantum disks in $\mathrm{ZB} \mathrm{GaAs}$ ) and to $\mathrm{I}_{1}$ stacking faults (i.e., $3 \mathrm{ML}$ thick $\mathrm{ZB}$ quantum disks in WZ GaAs) with the result of envelope function calculations carried out in the frame of the effective potential formalism [44]. As shown in Ref. [45], this method is well suited for calculating the energy of excitons in type II heterostructures. For simplicity, we use a one-band model for both the electron and hole. To account for the uniaxial compressive strain of $\varepsilon=-0.23 \%$ along the nanowire axis, we take for the electron in $\mathrm{ZB}$ and $\mathrm{WZ} \mathrm{GaAs} \mathrm{the} \mathrm{band} \mathrm{parameters}$ of the $\Gamma_{6}$ and $\Gamma_{8}$ bands, respectively, and for the hole those reported for the valence bands. While the band parameters are well known for ZB GaAs (we take the same parameters as those given in Ref. [40]), the $\Gamma_{8}-\mathrm{A}$ bandgap $\left(E^{\mathrm{WZ}}\right)$ is not known accurately. In addition, while it is accepted that the band alignment between WZ and ZB GaAs is type II, the exact values for the conduction and valence band offsets ( $\Delta C$ and $\Delta V$, respectively) are a matter of controversy. The effective mass for the $\Gamma_{8}$ conduction band in WZ GaAs has been predicted to be highly anisotropic $[38,46]$. To reproduce this anisotropy, we take the mass perpendicular to the nanowire axis $m_{\mathrm{e}}^{\mathrm{WZ}, \perp}=0.1 m_{0}$, while we vary the electron mass along the nanowire axis $\left(m^{\mathrm{WZ}, I \mid}\right)$ between 0.1 and $1 m_{0}$. In the absence of any clear consensus on the value of the effective mass of the A hole in WZ GaAs, we take it equal to $0.766 m_{0}$, as proposed in Ref. [14]. We note that it is undisputed that the WZ hole is heavy, and its precise mass does not significantly affect our calculations. In our simulations, we vary the values of $\Delta C$ and $m \mathrm{e}^{\mathrm{WZ}, I I}$, such that the PL energies computed for the crystal-phase quantum disks correspond to those measured experimentally. We emphasize that $E^{\mathrm{WZ}}$ is not a completely free parameter, as it is given by the sum of the excitonic bandgap of strained $\mathrm{WZ}$ GaAs (1.506 eV, see Fig. 5(d)) with the binding energy of the $\Gamma_{8}-\mathrm{A}$ exciton (which is easily obtained from $m \mathrm{e}^{\mathrm{WZ}, \perp}$ and $\left.m \mathrm{e}^{\mathrm{WZ}, I}\right)$. Similarly, $\Delta V$ is given by $E^{\mathrm{ZB}}+\Delta C-$ $E^{\mathrm{WZ}}$, where $E^{\mathrm{ZB}}$ is the bandgap of $\mathrm{ZB}$ GaAs with $\varepsilon=-0.23 \%$.

We first compute the energy of the exciton in the 3 ML thick ZB disk in WZ GaAs (i.e., the $\mathrm{I}_{1}$ stacking fault). As shown in Fig. 6(a), the electron localized in the $\mathrm{ZB}$ disk attracts the hole, forming a stable exciton. Interestingly, despite a type-II band alignment, we find that the modulus squared of the electron-hole overlap integral is $\left|\left\langle\Psi_{\mathrm{e}} \mid \Psi_{\mathrm{h}}\right\rangle\right|^{2}=0.25$, confirming that the radiative recombination of excitons in thin crystal phase quantum disks is efficient [45]. The spatial extent of the exciton wavefunction along the nanowire axis is $9 \mathrm{~nm}$, a value lower than the average distance between adjacent ZB disks in domain C (Fig. 4(c)). This result verifies a posteriori that the quantum disks in domain $\mathrm{C}$ are electronically uncoupled. Figure 6(b) shows the set of values of $\Delta C$ and $m_{\mathrm{e}}{ }^{\mathrm{WZ}, \mathrm{II}}$ giving an exciton energy of $1.492 \mathrm{eV}$, as measured experimentally. Upon increasing $\Delta C$ from 75 to $155 \mathrm{meV}, m e^{\mathrm{WZ}, I I}$ decreases from 1 to $0.1 m_{0}$. Remarkably, despite the large range of possible values for $\Delta C$ and $m \mathrm{e}^{\mathrm{WZ}, I I}$, possible values for $E^{\mathrm{WZ}}$ for WZ GaAs in the presence of a uniaxial strain of $\varepsilon=-0.23 \%$ range only between 1.513 and $1.519 \mathrm{eV}$.

So far, we have neglected the fact that WZ GaAs is a polar material with a spontaneous polarization field aligned along the $\langle 0001\rangle$ axis. As ZB GaAs is free of 
(a)
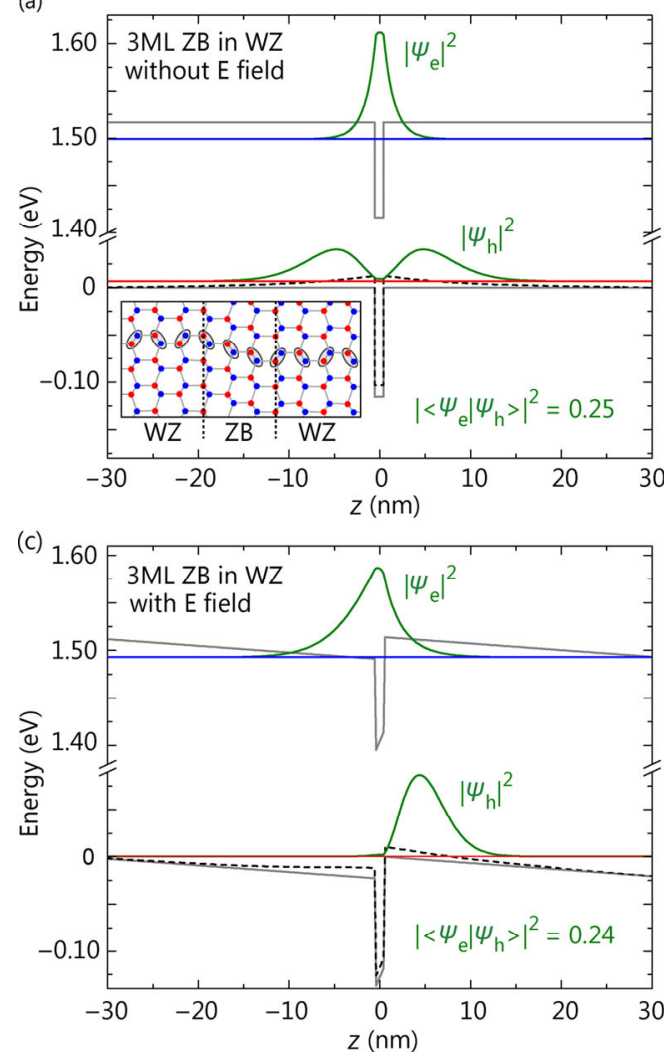

(b)

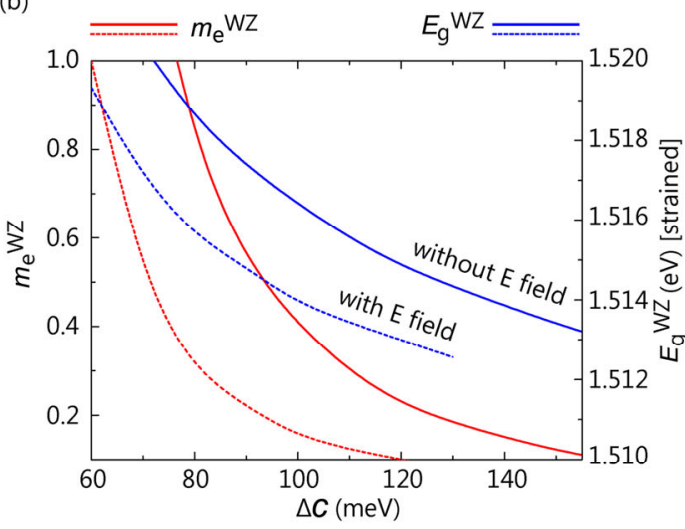

(d)

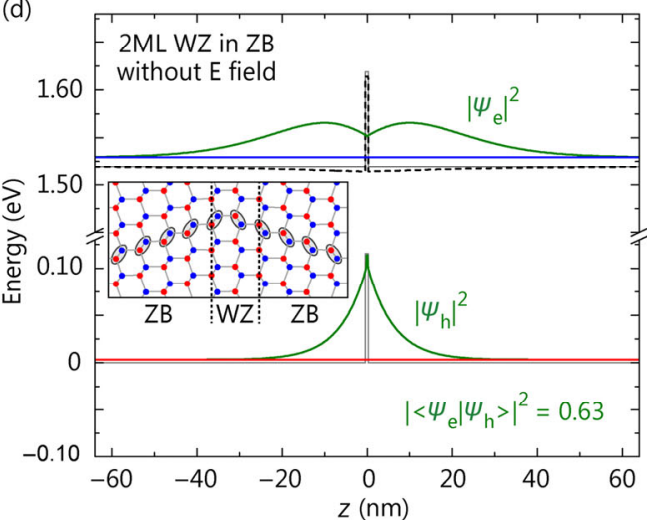

Figure 6 (a) Band profile (grey solid line), effective potential profile (black dashed line), envelope functions (green solid lines) and energy levels of electrons (blue) and holes (red) for excitons bound to a single 3-ML-thick ZB disk in WZ. The effective potential for the hole is the result of the sum of the crystal potential for the hole and the Coulomb potential created by the electron. The wave function has been calculated using $m_{\mathrm{e}}{ }^{\mathrm{WZ}}=0.4 m_{0}, E^{\mathrm{WZ}}=1.5164 \mathrm{eV}$ and $\Delta C=100 \mathrm{meV}$. Inset: model of the crystal structure showing the 3-ML-thick ZB inclusion between two WZ segments (Fig. 4(f)). The modulus squared of the electron-hole overlap integral $\left|<\Psi_{\mathrm{e}}\right| \Psi_{\mathrm{h}}>\left.\right|^{2}$ is also specified in the figure. (b) $m_{\mathrm{e}}{ }^{\mathrm{WZ}}$ (red line, left scale) and $E^{\mathrm{WZ}}$ (blue line, right scale) in a nanowire with $\varepsilon=-0.23 \%$ as a function of $\Delta C$, so that the computed energy of the exciton in a single 3-ML-thick ZB disk in the WZ is equal to $1.492 \mathrm{eV}$ (Fig. 4(d)). The solid and dashed lines show the result of the calculations without and with built-in electric fields, respectively. (c) Same as (a), but accounting for the built-in electric field induced by the discontinuities in the polarization fields at the interfaces between WZ and ZB GaAs. The wave function was calculated using $m_{\mathrm{e}}{ }^{\mathrm{WZ}}=0.15 m_{0}, E^{\mathrm{WZ}}=1.5139 \mathrm{eV}$ and $\Delta C=100 \mathrm{meV}$. (d) Band profile (grey solid line), effective potential profile (black dashed line), envelope functions (green solid lines), and energy levels of electron (blue) and hole (red) for excitons bound to a single 2-ML-thick WZ disk in the ZB matrix. The wave function has been calculated using $m_{\mathrm{e}}{ }^{\mathrm{WZ}}=0.4 m_{0}$. Inset: model of the crystal structure showing the 2-ML-thick WZ disk formed at the interface between two rotationally twinned ZB segments (Fig. 4(c)).

spontaneous polarization, the polarization field at the interfaces of crystal-phase quantum disks is discontinuous. It should be noted that this difference in the polarization at the interface will not change appreciably when including the piezoelectric contribution induced by the uniaxial strain, since the strain is most likely uniform and the piezoelectric properties of $\mathrm{ZB}$ and WZ GaAs are supposed to be very similar. As shown in Fig. 6(c), the built-in electric fields resulting from these discontinuities lead to a quantum-confined Stark effect that separates the electron and hole wavefunctions along the confinement axis [47]. For the $3 \mathrm{ML}$ thick ZB disks in WZ, however, only a minor change in PL energy is expected [45]. Accordingly, taking into account the spontaneous polarization of WZ GaAs (which we take equal to $2.7 \times 10^{-3} \mathrm{C} / \mathrm{m}^{2}$, see Ref. [48]) and using the same procedure as above, we find that $E^{\mathrm{WZ}}$ for $\mathrm{WZ}$ GaAs in the presence of a uniaxial strain of $\varepsilon=-0.23 \%$ ranges between 1.512 and $1.519 \mathrm{eV}$, a value close to that obtained when neglecting the polarization fields. Similarly, the value of $\left|\left\langle\Psi_{\mathrm{e}} \mid \Psi_{\mathrm{h}}\right\rangle\right|^{2}$ remains almost unchanged in the presence of built-in electric fields (Fig. 6(c)). In the actual PL experiment, built-in electric fields may be partially screened by photogenerated 
charge carriers. Therefore, the result of our calculations with and without polarization fields leads to the conclusion that the $\Gamma_{8}-\mathrm{A}$ bandgap in strain-free $\mathrm{WZ}$ GaAs is between 1.532 and $1.539 \mathrm{eV}$. For a strain-free WZ nanowire, however, we would expect the emission to originate not from the $\Gamma_{8}-\mathrm{A}$ but from the $\Gamma_{T} \mathrm{~A}$ transition. By extrapolating our data with the help of the strain variation reported by Signorello et al. [27], this transition would be located between 1.507 and $1.514 \mathrm{eV}$ in strain-free WZ GaAs.

Finally, we simulate the excitonic properties of $2 \mathrm{ML}$ thick WZ disks in ZB GaAs (i.e., twin boundaries) using the set of $\Delta C$ and $m \mathrm{e}^{\mathrm{wZ}, I I}$ values illustrated in Fig. 6(b). The spatial extent of the exciton wavefunction for the $2 \mathrm{ML}$ thick WZ disks is much larger than that computed for the $3 \mathrm{ML}$ thick ZB disks. For instance, for $\Delta C=100 \mathrm{meV}$ and $m_{\mathrm{e}}^{\mathrm{WZ}, I \mathrm{I}}=0.4 m_{0}$, the extent of the electron charge density along the nanowire axis is larger than $30 \mathrm{~nm}$ (Fig. 6(d)). Therefore, when the distance between adjacent quantum disks in domain A is about $15-20 \mathrm{~nm}$, they are electronically coupled. In other words, it is not easily possible to extract accurate values of $\Delta C$ and $m \mathrm{e}^{\mathrm{WZ}, 11}$ from the emission energy measured for the $2 \mathrm{ML}$ thick WZ disks in domain A.

\section{Conclusions}

In conclusion, we have correlated the emission and the structural properties of the $\mathrm{ZB}, \mathrm{WZ}$ and polytype segments in a GaAs nanowire at $10 \mathrm{~K}$. By combining the PL energies measured for the $\mathrm{WZ}$ and $\mathrm{ZB}$ segments as well as that from $3 \mathrm{ML}$ thick $\mathrm{ZB}$ disks in a WZ GaAs matrix with a simulation of the exciton energy in polytype nanowire segments, we obtained that the energy of the $\Gamma_{8}-\mathrm{A}$ free exciton in strain-free WZ $\mathrm{GaAs}$ at $10 \mathrm{~K}$ is $1.526 \mathrm{eV}$, and that the $\Gamma_{8}-\mathrm{A}$ bandgap energy ranges between 1.532 and $1.539 \mathrm{eV}$. Based on these results, and using the strain dependence reported in Ref. [27], we estimated that the $\Gamma_{T}-\mathrm{A}$ bandgap energy expected in strain-free nanowires is 1.507 to $1.514 \mathrm{eV}$. Our study revealed that the interaction between the Si supporting substrate and the dispersed nanowire can be strong enough to strain the latter. This strain results in energy shifts that may at least partly explain the discrepancy of the values reported for the bandgap of
WZ GaAs. Finally, as the energy difference between the $\Gamma_{7}$ and $\Gamma_{8}$ conduction bands is small, the symmetry of the fundamental conduction band of WZ GaAs is sensitive to strain and to radial confinement. In particular, we expect that for sufficiently small diameters, the fundamental conduction band of WZ GaAs nanowires is $\Gamma_{8}$ even in the absence of strain. Such thin GaAs nanowires with enhanced excitonic effects can be obtained by postgrowth thermal decomposition [49] and are of potential great interest for optoelectronic applications involving excitons.

\section{Methods}

\subsection{Molecular beam epitaxy}

In this work, $\mathrm{GaAs} /(\mathrm{Al}, \mathrm{Ga}) \mathrm{As}$ core/shell nanowires were grown under the same growth conditions used in previous work by Jahn et al. [9]. Molecular-beam epitaxy (MBE) was used to prepare nanowires in a self-catalyzed mode, i.e., Ga-assisted, vapor-liquid-solid (VLS) growth. The substrate selected for this growth was a phosphorous-doped on-axis $\left( \pm 1^{\circ}\right) \mathrm{Si}(111)$ wafer. To desorb the water from the surface, the wafer was heated under ultrahigh vacuum to $300{ }^{\circ} \mathrm{C}$ for $20 \mathrm{~min}$, while the native oxide layer remained on the substrate. Then, the substrate was heated to $580{ }^{\circ} \mathrm{C}$ and exposed to $\mathrm{A}_{\mathrm{S} 4}$ with a pressure of $1.2 \times 10^{-5} \mathrm{mbar}$. To initiate nanowire growth, the Ga shutter was opened, and the Ga flux was set to a value equivalent to a planar growth rate of $820 \mathrm{~nm} \cdot \mathrm{h}^{-1}$, corresponding to an $\mathrm{A}_{\mathrm{S}_{4}} / \mathrm{Ga}$ flux ratio of 1 . After $30 \mathrm{~min}$, the growth of an $(\mathrm{Al}, \mathrm{Ga}) \mathrm{As}$ shell with a nominal $\mathrm{Al}$ content of $10 \%$ was started. The resulting total growth time of $\mathrm{GaAs}-(\mathrm{Al}, \mathrm{Ga}) \mathrm{As}$ core-shell nanowires was $1 \mathrm{~h}$. The presence of a wide-bandgap shell efficiently suppresses nonradiative carrier recombination at the nanowire surface and hence increases the luminous efficiency, which is particularly important for the present study $[50,51]$. As a reference sample, a $1-\mu \mathrm{m}$-thick planar epitaxial layer of zincblende GaAs was grown using the same MBE system as for the nanowire fabrication.

\subsection{Experimental protocol}

In order to perform the cross-correlated NSOM and TEM study of the same crystal-phase quantum structures, 
GaAs nanowires were transferred from the growth substrate to a pre-patterned silicon supporting substrate. Particularly, Au alignment marks with an equidistant spacing of $25 \mu \mathrm{m}$ were structured on the Si surface by electron-beam lithography. Ultra-sonication in ethanol solution and drop-casting were used to detach nanowires from the growth substrate and disperse them on the substrate with alignment marks, respectively. At first, selected nanowires were studied by NSOM to exclude possible perturbations of the PL spectra by other characterization techniques. In the next step, the same nanowires investigated optically were prepared by FIB for structural analysis using electron microscopy. Thereby, the NSOM-TEM strategy allowed us to access the optical and structural properties of the same nanowire with a high spatial resolution. Finally, our experimental data were cross-correlated with simulations of the emission energy of the crystal-phase quantum structures to get an insight into the electronic properties of the WZ phase of GaAs.

\subsection{Near-field scanning optical microscopy}

For low-temperature near-field spectral imaging, the nanowire sample was fixed on the special holder of a continuous flow liquid-helium cryostat inside the NSOM vacuum chamber [52] and cooled down to 10 K. For the optical excitation, the 543-nm $(2.283 \mathrm{eV})$ line of a $0.5-\mathrm{mW}$ HeNe laser was used. The excitation power for single-nanowire imaging was adjusted using a linearly variable neutral density filter to insure a sufficient signal-to-noise ratio and at the same time avoid the perturbing effect of the high-power laser beam on the photoluminescence spectra, such as the shift of PL peak energy. Thus, a laser power of only $115 \mathrm{nW}$ was coupled to the single-mode optical fiber for near-field spectral imaging. A chemically etched tapered single-mode optical fiber was used as a near-field probe [53-55]. The PL signal was collected by the same probe operating in a near-field illumination-collection geometry [56], which typically provides a spatial resolution of about $1 / 4$ to $1 / 6$ of the working wavelength. The near-field probe-to-sample distance was kept constant during the scanning process using a shear-force distance control technique [57]. The collected photoluminescence signal was dispersed in a $0.5-\mathrm{m}$ focal length imaging monochromator. Its spectral resolution was set to $3 \mathrm{meV}$. Finally, the photoluminescence signal was recorded by an $\mathrm{LN}_{2}$-cooled (In,Ga)As charge coupled device camera. A schematic illustration of the single-nanowire NSOM experiment is depicted in our previous work [43]. For the spectral imaging, the fiber-probe was raster-scanned across the sample surface of $4 \times 4 \mu \mathrm{m}^{2}$ containing the nanowire of interest with a sampling step size, i.e., a spatial pixel, of $40 \mathrm{~nm}$. At every step, a full photoluminescence spectrum was recorded in the energy range from 1.244 to $1.872 \mathrm{eV}$, which covers the entire PL emission range expected for $\mathrm{GaAs} /(\mathrm{Al}, \mathrm{Ga})$ As nanowires.

\subsection{Transmission electron microscopy}

The crystal structure of the nanowire was then analyzed in a FEI microscope TITAN 80-300, which is equipped with a goniometer that allows the specimen to be tilted by up to $\pm 30^{\circ}$. This feature provides the degree of freedom required to reach the crystallographic orientation for structural analysis. The imaging of the crystal lattice structure was achieved by scanning transmission electron microscopy (STEM), especially applying high-angle annual dark field technique (HAADF). Lattice imaging was performed in the $\langle 110\rangle$ zone axis, in which the stacking sequence of consecutive $\{111\}$ lattice planes can be determined. This approach allowed us to distinguish between the $\mathrm{ZB}$ and $\mathrm{WZ}$ crystal phases. The entire length of each nanowire was investigated, providing both an overview of the nanowire morphology, planar defects, such as stacking faults, as well as the high-resolution information about the crystal structure, including the determination of the precise stacking sequence of the $\{111\}$ planes.

\section{Acknowledgements}

We thank Uwe Jahn for invaluable discussions, and Oliver Marquardt for a critical reading of the manuscript. A. S. is indebted to Christoph Lienau for fruitful discussions of NSOM experiments, Horst Blumtritt for the FIB preparation of single nanowires for TEM analysis, as well as to Wilfried Erfurth and Kornelia Sklarek for electron-beam lithography. P. C. acknowledges funding from the Fonds National Suisse de la Recherche Scientifique through project No. 161032. 
Funding: Open access funding provided by Max Planck Society.

Electronic Supplementary Material: Supplementary material (survey on the band gap energy of wurtzite GaAs, photoluminescence of reference GaAs planar epitaxial layer measured by NSOM, composition analysis of $\mathrm{GaAs} /(\mathrm{Al}, \mathrm{Ga})$ As core/shell nanowires, and strain analysis by Raman spectroscopy measurements) is available in the online version of this article at https://doi.org/10.1007/s12274-018-2053-5.

Open Access: This article is distributed under the terms of the Creative Commons Attribution 4.0 International License (http://creativecommons.org/licenses/by/4.0/), which permits unrestricted use, distribution, and reproduction in any medium, provided you give appropriate credit to the original author(s) and the source, provide a link to the Creative Commons license, and indicate if changes were made.

\section{References}

[1] Caroff, P.; Bolinsson, J.; Johansson, J. Crystal phases in III-V nanowires: From random toward engineered polytypism. IEEE J. Sel. Top. Quantum Electron. 2011, 17, 829-846.

[2] Glas, F.; Harmand, J. C.; Patriarche, G. Why does wurtzite form in nanowires of III-V zinc blende semiconductors? Phys. Rev. Lett. 2007, 99, 146101.

[3] Dubrovskii, V. G.; Sibirev, N. V.; Harmand, J. C.; Glas, F. Growth kinetics and crystal structure of semiconductor nanowires. Phys. Rev. B 2008, 78, 235301.

[4] Krogstrup, P.; Jørgensen, H. I.; Johnson, E.; Madsen, M. H.; Sørensen, C. B.; Fontcuberta i Morral, A.; Aagesen, M.; Nygård, J.; Glas, F. Advances in the theory of III-V nanowire growth dynamics. J. Phys. D: Appl. Phys. 2013, 46, 313001.

[5] Jacobsson, D.; Panciera, F.; Tersoff, J.; Reuter, M. C.; Lehmann, S.; Hofmann, S.; Dick, K. A.; Ross, F. M. Interface dynamics and crystal phase switching in GaAs nanowires. Nature 2016, 531, 317-322.

[6] Bechstedt, F.; Belabbes, A. Structure, energetics, and electronic states of III-V compound polytypes. J. Phys.: Condens. Matter 2013, 25, 273201.

[7] Bao, J. M.; Bell, D. C.; Capasso, F.; Wagner, J. B.; Mårtensson, T.; Trägårdh, J.; Samuelson, L. Optical properties of rotationally twinned InP nanowire heterostructures. Nano Lett. 2008, 8, 836-41.
[8] Spirkoska, D.; Arbiol, J.; Gustafsson, A.; Conesa-Boj, S.; Glas, F.; Zardo, I.; Heigoldt, M.; Gass, M. H.; Bleloch, A. L.; Estrade, S. et al. Structural and optical properties of high quality zinc-blende/wurtzite GaAs nanowire heterostructures. Phys. Rev. B 2009, 80, 245325.

[9] Jahn, U.; Lähnemann, J.; Pfüller, C.; Brandt, O.; Breuer, S.; Jenichen, B.; Ramsteiner, M.; Geelhaar, L.; Riechert, H. Luminescence of GaAs nanowires consisting of wurtzite and zinc-blende segments. Phys. Rev. B 2012, 85, 045323.

[10] Thelander, C.; Caroff, P.; Plissard, S.; Dey, A. W.; Dick, K. A. Effects of crystal phase mixing on the electrical properties of InAs nanowires. Nano Lett. 2011, 11, 2424-2429.

[11] Bussone, G.; Schäfer-Eberwein, H.; Dimakis, E.; Biermanns, A.; Carbone, D.; Tahraoui, A.; Geelhaar, L.; Haring Bolívar, P.; Schülli, T. U.; Pietsch, U. Correlation of electrical and structural properties of single as-grown GaAs nanowires on $\mathrm{Si}(111)$ substrates. Nano Lett. 2015, 15, 981-989.

[12] Tersoff, J. Stable self-catalyzed growth of III-V nanowires. Nano Lett. 2015, 15, 6609-6613.

[13] Dubrovskii, V. G. Development of growth theory for vaporliquid-solid nanowires: Contact angle, truncated facets, and crystal phase. Cryst. Growth Des. 2017, 17, 2544-2548.

[14] Heiss, M.; Conesa-Boj, S.; Ren, J.; Tseng, H. H.; Gali, A.; Rudolph, A.; Uccelli, E.; Peiró, F.; Morante, J. R.; Schuh, D. et al. Direct correlation of crystal structure and optical properties in wurtzite/zinc-blende GaAs nanowire heterostructures. Phys. Rev. B 2011, 83, 045303.

[15] Ketterer, B.; Heiss, M.; Livrozet, M. J.; Rudolph, A.; Reiger, E.; Fontcuberta I Morral, A. Determination of the band gap and the split-off band in wurtzite GaAs using Raman and photoluminescence excitation spectroscopy. Phys. Rev. B 2011, 83, 125307.

[16] Ahtapodov, L.; Todorovic, J.; Olk, P.; Mjåland, T.; Slåttnes, P.; Dheeraj, D. L.; van Helvoort, A. T. J.; Fimland, B. O.; Weman, H. A story told by a single nanowire: Optical properties of wurtzite GaAs. Nano Lett. 2012, 12, 6090-6095.

[17] Hjort, M.; Lehmann, S.; Knutsson, J.; Timm, R.; Jacobsson, D.; Lundgren, E.; Dick, K. A.; Mikkelsen, A. Direct imaging of atomic scale structure and electronic properties of GaAs wurtzite and zinc blende nanowire surfaces. Nano Lett. 2013, 13, 4492-4498.

[18] Kim, D. C.; Dheeraj, D. L.; Fimland, B. O.; Weman, H. Polarization dependent photocurrent spectroscopy of single wurtzite GaAs/AlGaAs core-shell nanowires. Appl. Phys. Lett. 2013, 102, 142107.

[19] Martelli, F.; Priante, G.; Rubini, S. Photoluminescence of GaAs nanowires at an energy larger than the zincblende band-gap: Dependence on growth parameters. Semicond. Sci. Technol. 2015, 30, 055020 . 
[20] Mukherjee, A.; Ghosh, S.; Breuer, S.; Jahn, U.; Geelhaar, L.; Grahn, H. T. Spatially resolved study of polarized microphotoluminescence spectroscopy on single GaAs nanowires with mixed zincblende and wurtzite phases. J. Appl. Phys. 2015, 117,054308

[21] Vainorius, N.; Lehmann, S.; Jacobsson, D.; Samuelson, L.; Dick, K. A.; Pistol, M. E. Confinement in thickness-controlled GaAs polytype nanodots. Nano Lett. 2015, 15, 2652-2656.

[22] Vainorius, N.; Lehmann, S.; Gustafsson, A.; Samuelson, L.; Dick, K. A.; Pistol, M. E. Wurtzite GaAs quantum wires: One-dimensional subband formation. Nano Lett. 2016, 16, 2774-2780.

[23] Loitsch, B.; Müller, M.; Winnerl, J.; Veit, P.; Rudolph, D.; Abstreiter, G.; Finley, J. J.; Bertram, F.; Christen, J.; Koblmüller, G. Microscopic nature of crystal phase quantum dots in ultrathin GaAs nanowires by nanoscale luminescence characterization. New J. Phys. 2016, 18, 063009.

[24] Timofeeva, M.; Bouravleuv, A.; Cirlin, G.; Shtrom, I.; Soshnikov, I.; Reig Escalé, M.; Sergeyev, A.; Grange, R. Polar second-harmonic imaging to resolve pure and mixed crystal phases along GaAs nanowires. Nano Lett. 2016, 16, 6290-6297.

[25] McMahon, M. I.; Nelmes, R. J. Observation of a wurtzite form of gallium arsenide. Phys. Rev. Lett. 2005, 95, 215505.

[26] Gustafsson, A.; Bolinsson, J.; Sköld, N.; Samuelson, L. Determination of diffusion lengths in nanowires using cathodoluminescence. Appl. Phys. Lett. 2010, 97, 072114.

[27] Signorello, G.; Lörtscher, E.; Khomyakov, P. A.; Karg, S.; Dheeraj, D. L.; Gotsmann, B.; Weman, H.; Riel, H. Inducing a direct-to-pseudodirect bandgap transition in wurtzite GaAs nanowires with uniaxial stress. Nat. Commun. 2014, 5, 3655.

[28] Bogardus, E. H.; Bebb, H. B. Bound-exciton, free-exciton, band-acceptor, donor-acceptor, and auger recombination in GaAs. Phys. Rev. 1968, 176, 993-1002.

[29] Shah, J.; Leite, R. C. C.; Nahory, R. E. Photoluminescence and photoconductivity in undoped epitaxial GaAs. Phys. Rev. 1969, 184, 811-820.

[30] Hiruma, K.; Yazawa, M.; Katsuyama, T.; Ogawa, K.; Haraguchi, K.; Koguchi, M.; Kakibayashi, H. Growth and optical properties of nanometer-scale GaAs and InAs whiskers. J. Appl. Phys. 1995, 77, 447.

[31] Hestroffer, K.; Mata, R.; Camacho, D.; Leclere, C.; Tourbot, G.; Niquet, Y. M.; Cros, A.; Bougerol, C.; Renevier, H.; Daudin, B. The structural properties of GaN/AlN core-shell nanocolumn heterostructures. Nanotechnology 2010, 21, 415702.

[32] Hocevar, M.; Thanh Giang, L. T.; Songmuang, R.; den Hertog, M.; Besombes, L.; Bleuse, J.; Niquet, Y. M.; Pelekanos, N. T. Residual strain and piezoelectric effects in passivated GaAs/ AlGaAs core-shell nanowires. Appl. Phys. Lett. 2013, 102, 191103.
[33] Schlager, J. B.; Bertness, K. A.; Blanchard, P. T.; Robins, L. H.; Roshko, A.; Sanford, N. A. Steady-state and time-resolved photoluminescence from relaxed and strained GaN nanowires grown by catalyst-free molecular-beam epitaxy. J. Appl. Phys. 2008, 103, 124309.

[34] Corfdir, P.; Feix, F.; Zettler, J. K.; Fernández-Garrido, S.; Brandt, O. Importance of the dielectric contrast for the polarization of excitonic transitions in single $\mathrm{GaN}$ nanowires. New J. Phys. 2015, 17, 033040.

[35] Stan, G.; Krylyuk, S.; Davydov, A. V.; Cook, R. F. Bending manipulation and measurements of fracture strength of silicon and oxidized silicon nanowires by atomic force microscopy. J. Mater. Res. 2012, 27, 562-570.

[36] Gershoni, D.; Henry, C. H.; Baraff, G. A. Calculating the optical properties of multidimensional heterostructures: Application to the modeling of quaternary quantum well lasers. IEEE $J$. Quantum Electron. 1993, 29, 2433-2450.

[37] Cheiwchanchamnangij, T.; Lambrecht, W. R. L. Band structure parameters of wurtzite and zinc-blende GaAs under strain in the GW approximation. Phys. Rev. B 2011, 84, 035203.

[38] Belabbes, A.; Panse, C.; Furthmüller, J.; Bechstedt, F. Electronic bands of III-V semiconductor polytypes and their alignment. Phys. Rev. B 2012, 86, 075208.

[39] Greil, J.; Assali, S.; Isono, Y.; Belabbes, A.; Bechstedt, F.; Valega Mackenzie, F. O.; Silov, A. Y.; Bakkers, E. P. A. M.; Haverkort, J. E. M. Optical properties of strained wurtzite gallium phosphide nanowires. Nano Lett. 2016, 16, 3703-3709.

[40] Corfdir, P.; Van Hattem, B.; Uccelli, E.; Conesa-Boj, S.; Lefebvre, P.; Fontcuberta I Morral, A.; Phillips, R. T. Threedimensional magneto-photoluminescence as a probe of the electronic properties of crystal-phase quantum disks in $\mathrm{GaAs}$ nanowires. Nano Lett. 2013, 13, 5303-5310.

[41] Algra, R. E.; Verheijen, M. A.; Borgström, M. T.; Feiner, L. F.; Immink, G.; van Enckevort, W. J. P.; Vlieg, E.; Bakkers, E. P. A. M. Twinning superlattices in indium phosphide nanowires. Nature 2008, 456, 369-372.

[42] Rudolph, D.; Schweickert, L.; Morkötter, S.; Hanschke, L.; Hertenberger, S.; Bichler, M.; Koblmüller, G.; Abstreiter, G.; Finley, J. J. Probing the trapping and thermal activation dynamics of excitons at single twin defects in GaAs-AlGaAs core-shell nanowires. New J. Phys. 2013, 15, 113032.

[43] Senichev, A. V.; Talalaev, V. G.; Shtrom, I. V.; Blumtritt, H.; Cirlin, G. E.; Schilling, J.; Lienau, C.; Werner, P. Nanospectroscopic imaging of twinning superlattices in an individual GaAs-AlGaAs core-shell nanowire. ACS Photonics 2014, 1, 1099-1106.

[44] Bellabchara, A.; Lefebvre, P.; Christol, P.; Mathieu, H. Improved modeling of excitons in type-II semiconductor heterostructures by use of a three-dimensional variational function. Phys. Rev. B 1994, 50, 11840-11844. 
[45] Corfdir, P.; Lefebvre, P. Importance of excitonic effects and the question of internal electric fields in stacking faults and crystal phase quantum discs: The model-case of GaN. J. Appl. Phys. 2012, 112, 053512.

[46] De, A.; Pryor, C. E. Predicted band structures of III-V semiconductors in the wurtzite phase. Phys. Rev. B 2010, 81, 155210.

[47] Karin, T.; Linpeng, X.; Glazov, M. M.; Durnev, M. V.; Ivchenko, E. L.; Harvey, S.; Rai, A. K.; Ludwig, A.; Wieck, A. D.; Fu, K. M. C. Giant permanent dipole moment of two-dimensional excitons bound to a single stacking fault. Phys. Rev. B 2016, 94, 41201.

[48] Bauer, B.; Hubmann, J.; Lohr, M.; Reiger, E.; Bougeard, D.; Zweck, J. Direct detection of spontaneous polarization in wurtzite GaAs nanowires. Appl. Phys. Lett. 2014, 104, 211902.

[49] Loitsch, B.; Rudolph, D.; Morkötter, S.; Döblinger, M.; Grimaldi, G.; Hanschke, L.; Matich, S.; Parzinger, E.; Wurstbauer, U.; Abstreiter, G. et al. Tunable quantum confinement in ultrathin, optically active semiconductor nanowires via reverse-reaction growth. Adv. Mater. 2015, 27, 2195-2202.

[50] Titova, L. V.; Hoang, T. B.; Jackson, H. E.; Smith, L. M.; Yarrison-Rice, J. M.; Kim, Y.; Joyce, H. J.; Tan, H. H.; Jagadish, C. Temperature dependence of photoluminescence from single core-shell GaAs-AlGaAs nanowires. Appl. Phys. Lett. 2006, 89, 173126.

[51] Tomioka, K.; Kobayashi, Y.; Motohisa, J.; Hara, S.; Fukui, T.
Selective-area growth of vertically aligned GaAs and GaAs/ AlGaAs core-shell nanowires on $\mathrm{Si}(111)$ substrate. Nanotechnology 2009, 20, 145302.

[52] Behme, G.; Richter, A.; Süptitz, M.; Lienau, C. Vacuum near-field scanning optical microscope for variable cryogenic temperatures. Rev. Sci. Instrum. 1997, 68, 3458-3463.

[53] Intonti, F.; Emiliani, V.; Lienau, C.; Elsaesser, T.; Savona, V.; Runge, E.; Zimmermann, R.; Nötzel, R.; Ploog, K. H. Quantum mechanical repulsion of exciton levels in a disordered quantum well. Phys. Rev. Lett. 2001, 87, 076801.

[54] Emiliani, V.; Guenther, T.; Lienau, C.; Nötzel, R.; Ploog, K. H. Ultrafast near-field spectroscopy of quasi-one-dimensional transport in a single quantum wire. Phys. Rev. B 2000, 61, R10583-R10586.

[55] Guenther, T.; Lienau, C.; Elsaesser, T.; Glanemann, M.; Axt, V. M.; Kuhn, T.; Eshlaghi, S.; Wieck, A. D. Coherent nonlinear optical response of single quantum dots studied by ultrafast near-field spectroscopy. Phys. Rev. Lett. 2002, 89, 057401.

[56] Intonti, F.; Emiliani, V.; Lienau, C.; Elsaesser, T.; Nötzel, R.; Ploog, K. H. Near-field optical spectroscopy of localized and delocalized excitons in a single GaAs quantum wire. Phys. Rev. $B$ 2001, 63, 075313.

[57] Karrai, K.; Grober, R. D. Piezoelectric tip-sample distance control for near field optical microscopes. Appl. Phys. Lett. 1995, 66, $1842-1844$. 